\title{
Routing Protocol for Wireless Sensor Network Based on Automatic Cluster Optimization
}

\author{
https://doi.org/10.3991/ijoe.v14i12.9297 \\ Hu Zhongdong ${ }^{\bowtie}$, Wang Zhendong, Wang Junling, Zeng Ting \\ Jiangxi University of Science and Technology, Ganzhou, China \\ jxhzd@163.com
}

\begin{abstract}
The existing routing protocols such as LEACH and its improvements balance the overall energy consumption of the network by electing cluster heads looping and randomly. However, it also causes defects such as different cluster sizes and uneven cluster head distribution, resulting in uneven energy loss. In response to these shortcomings, this paper proposes a new routing protocol called AOCRP based on automatic optimization of clustering. The AOCRP has an optimized and fixed number of clusters, and the cluster head distribution is relatively uniform. It can adjust the number of nodes in each cluster automatically during the operation and maintain the number of nodes the same in each cluster during each round. The AOCRP balances node energy loss and extends the life cycle of wireless sensor networks. Simulation experiments show that the performance of this protocol is better than EH-LEACH and DEEC in extending the network lifecycle and data transmission.
\end{abstract}

Keywords - wireless sensor network, AOCRP, energy heterogeneity, optimized clustering, cluster head election mechanism

\section{Introduction}

Routing protocols are important foundation for wireless sensor network (Wireless Sensor Network, WSN) applications. In wireless sensor network applications, routing protocols are critical for the monitoring activities of sensor networks. In the environmental monitoring, such as forest fire, geological disaster monitoring and early warning, the monitoring data of the sensor nodes must be transmitted to the control center[12]. Due to the energy limitation of micro-sensor nodes and the dense deployment of WSN and unattended application characteristics, it is difficult for nodes to add energy. These factors determine the primary way to extend the WSN life time is to balance energy consumption efficiently.[3-5]. Therefore, it is important to study efficient and low-energy wireless sensor network routing protocols.

Classification from the perspective of network topology, WSN routing protocols can be divided into planar routing protocols and hierarchical (clustered) routing protocols. Because the plane routing protocol needs to maintain a large routing table and no data fusion during operation, the node consumes a large amount of energy and is not suitable 
for use in large-scale networks. The clustering routing protocol adopts data fusion technologies. Before the data is sent to the base station, the cluster head is used to fuse and compress the data sent by the common node, which greatly reduces the energy consumption of the node. The LEACH (Low Energy Adaptive Clustering Hierarchy) protocol is the most representative hierarchical routing protocol. Heinzelman et al. first proposed a clustering routing protocol, which is a representative of the classical clustering routing protocol [6-7]. Since then, many scholars have improved and optimized on this basis from different levels. Such as Huang Lixiao, Wang Hui, Yuan Liyong et al. proposed an LEACH-improved protocol algorithm for WSN based on energy load balancing [8]. The DEEC protocol was proposed by Qing L, Zhu Q X, Wang M W[9]. An improved energy heterogeneous clustering routing algorithm was proposed by $\mathrm{Li}$ Anchao and Chen Guifen[10]. Gupta S K, Kuila P, Wei C J, and Wang W have optimized and improved cluster-based routing protocols [11-14].

LEACH and a series of improved clustering routing protocols are all based on the cyclical random election of cluster heads to balance the overall energy consumption of the network, However, they also have many defects such as the cluster head distribution may be uneven, and there may be too many or too few cluster heads, the number of nodes in each cluster is quite different, and the energy loss is uneven. In response to these shortcomings, a new routing protocol for wireless sensor network based on automatic cluster optimization (Routing Protocol for Wireless Sensor Network Based on Automatic Cluster Optimization, AOCRP) is proposed. The protocol has an optimized and fixed number of clusters, can adjust the number of nodes in each cluster during operation automatically to make the number of nodes of each cluster the same, balances the node energy loss, and prolongs the life cycle of the WSN.

In this paper, the development and main defects of the clustering routing protocol are introduced in the first section. The principle of the EH-LEACH (Energy Heterogeneous Low Energy Adaptive Clustering Hierarchy, EH-LEACH) protocol is described in Section 2, which is a classic LEACH(Low Energy Adaptive Clustering Hierarchy, LEACH) protocol with different energy levels for sensor nodes. The principle of the DEEC routing protocol is discussed in Section 3, which is a clustered routing protocol based on the LEACH routing protocol. The principle and implementation of the AOCRP routing protocol are proposed in Section 4. Simulation experiments and comparative analysis of EH-LEACH routing protocol, DEEC routing protocol and AOCRP routing protocol are expressed in Section 5. Through theoretical analysis and simulation experiments, it is concluded that the AOCRP routing protocol is better than the EH-LEACH protocol and the DEEC routing protocol in Section 6.

\section{EH-LEACH routing protocol}

The Energy Heterogeneous Low Energy Adaptive Clustering Hierarchy (EHLEACH) mentioned in this paper is the same as the LEACH protocol except that the energy of the sensor nodes is different. Each node becomes a cluster head node randomly in a cyclic manner. The data is merged at the cluster head and transmitted to the sink node. The EH-LEACH protocol has the advantages of hierarchical cluster structure 
and dynamic allocation of cluster head nodes. Especially when dealing with highly correlated data, most of the redundant data can be eliminated, therefore, it has better performance in terms of energy consumption. The threshold function for selecting a node to become the cluster head is set to

$$
T(n)=\left\{\begin{array}{c}
\frac{p}{1-p\left(\operatorname{rmod} \frac{1}{p}\right)}, \text { if } n \in G \\
0, \text { otherwise }
\end{array}\right.
$$

Where $p$ is the average probability that the node will be elected as a cluster head, $r$ represents the current number of running rounds, $G$ denotes the set of nodes that the node still fails to serve as the cluster head after $1 / p$ round. $\mathrm{r}$ mod $1 / p$ indicates the number of nodes that have not been elected to the cluster head. The node $\mathrm{n}_{i}$ generates a random number between 0 and 1 as a probability threshold to determine whether to be elected as a cluster head. In each round of cluster head election process, there may be too many or too few cluster heads, and the cluster heads are distributed unevenly, which is likely to be concentrated in a certain area, which will cause the energy consumption of the cluster head nodes to be larger.

\section{DEEC routing protocol}

The DEEC (Distributed Energy-Efficient Clustering algorithm, DEEC)[9] routing protocol is a multi-level clustering routing protocol based on the LEACH routing protocol. The nodes in each cluster are elected as cluster heads in turn. Since the initial energy of each node is different, the probability that the nodes become cluster heads is different. The initial energy and residual energy of the node are considered by the DEEC routing protocol, which relatively prolongs the life cycle of the network. If the $\mathrm{N}$ sensor nodes are deployed in the test area evenly, the ratio of the number of cluster heads to the total number of nodes is $p_{\text {opt }}$, then the probability for the node to become a cluster head is

$$
p_{i}=p_{o p t}
$$

Where $E_{r}(i)$ is the remaining energy of node $i$ in the $r$-th wheel, $\bar{E}(r)$ is the average remaining energy of the current network.

The threshold function of the node $s_{i}$ in each round selected as the cluster head in the DEEC routing protocol is:

$$
T\left(s_{i}\right)=\left\{\begin{array}{c}
\frac{p_{i}}{1-p_{i}\left(r \bmod \frac{1}{p_{i}}\right)}, \text { if } s_{i} \in G \\
0, \text { otherwise }
\end{array}\right.
$$

Where $G$ is a set of $1 / p_{i}$ rounds that are not selected as cluster head nodes; $P_{i}$ is the probability that the node $s_{i}$ is selected as the cluster head, $r$ is the current number of rounds. 
The DEEC routing protocol is improved on the basis of the $\mathrm{LEACH}$ routing protocol. The main part of the improvement is to consider the residual energy of the node when selecting the cluster head, but does not consider the influence of the location of the cluster head node. Compared with the LEACH routing protocol, the energy consumption of the cluster head node of the DEEC protocol is more balanced. However, the problem of excessive or too few cluster heads and uneven cluster head distribution has not been solved.

\section{$4 \quad$ AOCRP routing protocol}

\subsection{Network model}

It is assumed that $N$ sensor nodes are randomly distributed in a square area of $M \times M$ or a rectangular area of $L \times M$, and the environment is continuously monitored.

1. The sensor network is a static network, and the sensor nodes and base stations are no longer moved after deployment and the node locations are known. Each node has a global unique $i d$ identifier.

2. All nodes are energy isomerized, and their energy is limited and cannot be replenished;

3. Each node has the same function and has the ability to fuse data;

4. The base station is located outside the observation area, and the energy is not limited;

5. The wireless transmit power of the node is controllable, that is, the node can adjust its transmit power according to the distance of the receiver;

6. The network nodes are all duplex communication and can communicate directly with the base station. The energy consumed from node $i$ to node $j$ is equivalent to the energy consumed from node $j$ to node $i$.

\subsection{Energy consumption model}

The communication model is the same as the first-order radio model in the LEACH protocol, As shown in Figure 1. 


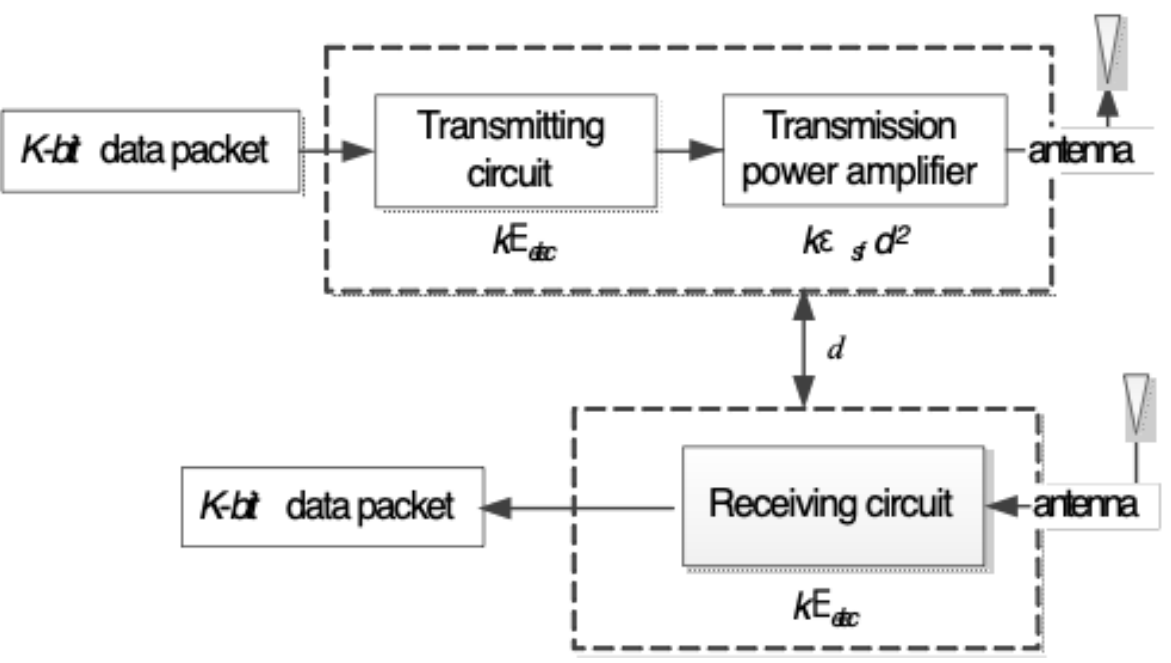

Fig. 1. Radio communication model

The energy consumption for sending a $k$-bit packet to a target node of distance $d$ is[15]:

$$
E_{T X}(k, d)=\left\{\begin{array}{l}
k E_{\text {elec }}+k \varepsilon_{f s} d^{2} d<d_{0} \\
k E_{\text {elec }}+k \varepsilon_{m p} d^{4} d>d_{0}
\end{array}\right.
$$

Where $E_{T X}$ is the energy consumption for transmitting data, $E_{\text {elec }}$ represents the energy consumption of the circuit portion when the sensor node transmits or receives 1 bit of data. When the transmission distance $d$ of the signal is less than a certain threshold $d_{0}$, the transmission energy is calculated according to the Free Space Model. The energy consumption value of the transmission power amplifier is proportional to the square of the transmission distance $d$; When the transmission distance $d$ of the signal is greater than the threshold $d_{0}$, the transmission energy consumption is calculated according to the Multi Paths Model. The power consumption value of the transmission power amplifier is proportional to the fourth power of the transmission distance $d$. Among them,

$\varepsilon_{f s}$ and $\varepsilon_{m p}$ are the energy consumption parameters of the transmission power amplifier under the above two energy consumption models respectively. The critical value $d_{0}$ can be calculated as follows:

$$
d_{0}=\sqrt{\frac{\varepsilon_{f s}}{\varepsilon_{m p}}}
$$

The energy consumed by the receiving circuit to receive $\mathrm{k}$ bit data is calculated as follows:

$$
E_{R X}(k)=k E_{\text {elec }}
$$




\subsection{Optimal clustering}

The number of cluster heads in the clustering routing protocol has a great impact on the node's energy consumption and network life cycle. According to the literature [16], the optimal number of cluster heads can be found as:

$$
k_{o p t}=\frac{\sqrt{N \cdot \varepsilon_{f s}}}{\sqrt{2 \pi \cdot \varepsilon_{m p}}} \cdot \frac{M}{d_{t o B S}^{2}}
$$

Where $\varepsilon_{f s}$ and $\varepsilon_{m p}$ are the energy consumption parameters of the transmission power amplifier under the two energy consumption models of the free space channel model and the multipath fading channel model. $N$ is the number of nodes, $M$ is the length of the area, and $d_{t o B S}$ is the distance from the node to the base station. The optimal cluster head number $k_{\text {opt }}$ is related to the coverage area $M^{2}$ of the node, the total number of nodes $N$, and the distance from the node to the base station. When $N=100, M=100 \mathrm{~m}$, the distance between the base station and the nodes in the network ranges from $75 m<d_{t o B s}<185 m$. It can be seen that the optimal cluster head number ranges from $1<k_{\text {opt }}<6$. It is also possible to determine the optimal number of clusters more accurately through simulation experiments.

\subsection{Cluster establishment phase}

1. Clustering: After determining the optimal cluster number $\mathrm{k}$ of the entire measurement area of the wireless sensor network, the base station divides the measurement area into k cluster areas evenly. The base station sends the coordinate range of each cluster area to all nodes. The node determines the cluster according to its coordinates, and sends the cluster number, node energy and coordinates to the base station.

2. Adjust the number of nodes in the cluster area: The base station counts the number of nodes in each cluster area and adjusts the number of nodes in each cluster area to be consistent. In addition, calculate the sum of the average initial energy of all nodes in each cluster area, and the average distance from all nodes in the cluster area to the base station and send finally it back to all nodes.

3. Cluster head election: In the first round, the base station specifies the energy maximum node of each cluster area as the cluster head, and thus each cluster area has a cluster head. At the beginning of the second round, each node transmits the last function of the next round of the cluster function, and the value of the election function $\mathrm{f}(\mathrm{i})$, which is the next round of the cluster head, is transmitted to the cluster head together with the observation data and the remaining energy value of the node. The data is then fused and compressed at the cluster head, and the compressed data along with the number of nodes of the cluster are transmitted to the base station.

Assume the calculation of the cluster head election function $f(i)$ of node $i$ running in $r$ round, cluster area $j$ :

$$
f(i)=a \cdot \frac{E_{i r}}{E_{0 j a v r}}+(1-a) \cdot \frac{\left(R_{\text {jmax }}-R_{\text {itos }}\right)}{R_{\text {javr }}}
$$


Where: $E_{i r}$ is the residual energy of node $i$ in r-round, $E_{0 j a v r}$ is the initial average energy of all nodes in cluster $j$, Rjmax is the maximum distance from node to base station of cluster $j, R_{i t o s}$ is the distance from node $i$ to base station, $R_{j a v r}$ is the average value of the distance from the node to the base station of the cluster area $j, a$ is the balance factor of the node energy and the distance, and the value of $a$ is 0.1 to 0.9 . The smaller the value of $a$, the greater the distance from the node to the base station; the larger the value of $a$, the greater the residual energy of the node. It can be seen from the simulation experiments that the balance factor $a$ has a value of 0.5 to 0.7 . Each node calculates its own $f(i)$ value and sends it to the cluster head. Each current cluster head selects the node with the maximum value of $f(i)$ in each cluster area as the cluster head of the next round according to the received election function $f(i)$ value, and broadcasts to the entire cluster, and proceeds to the next round of data transmission.

\section{5 data transmission phase}

After the intra-cluster node receives the CDMA(Code Division Multiple Access, CDMA) coding mode and TDMA(Time Division Multiple Address, TDMA) time slot allocated by the cluster head, the AOCRP routing protocol enters the stable data transmission phase. The environmental monitoring data is continuously acquired by the node, and the task of transmitting data to the cluster head node is completed within a given TDMA time slot. In order to reduce the energy consumption of the node, after the data is transmitted, the node turns off the signal transmitter and enters a sleep state. The data fusion process is performed at the cluster head node and the processed data is transmitted to the base station.

\section{$5 \quad$ Results}

\subsection{Simulation parameter settings}

The simulation experiments of EH-LEACH routing protocol, DEEC routing protocol and AOCRP routing protocol were carried out using MATLAB R2014a simulation software. The simulation scene is a rectangular area of $120 \mathrm{~m} \times 80 \mathrm{~m}$. The total number of nodes is $\mathrm{N}=120$, the nodes are randomly distributed, and the base station (or Sink) is located $80 \mathrm{~m}$ directly above the area, as shown in Figure 2. The simulation parameters are shown in Table 1. 


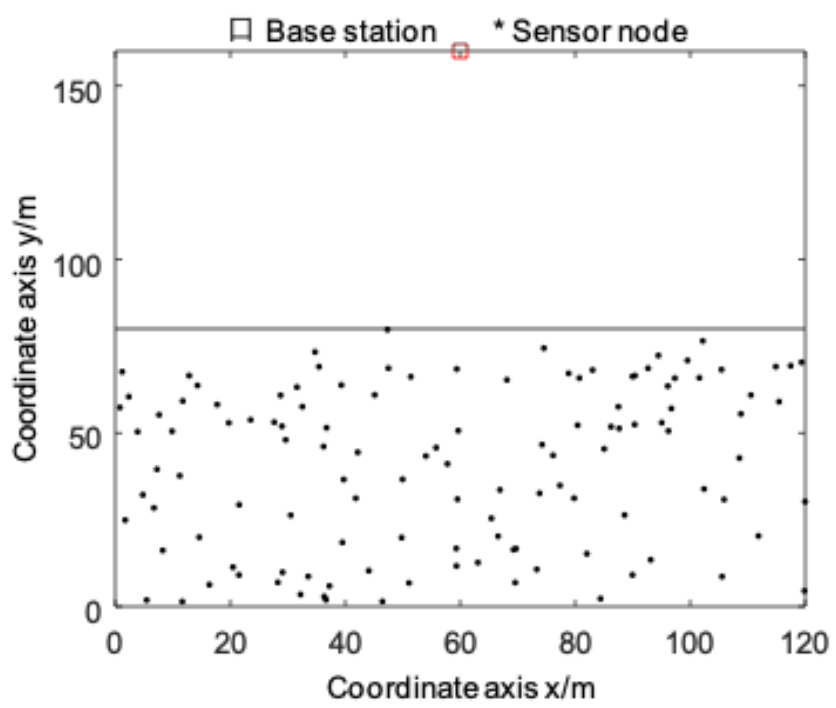

Fig. 2. Schematic diagram of random distribution of sensor nodes

Table 1. Simulation parameters

\begin{tabular}{|l|c|}
\hline \multicolumn{1}{|c|}{ parameter name } & parameter value \\
\hline Network range $/ \mathrm{m}$ & $(0,0) \sim(120,80)$ \\
\hline Sink location $/ \mathrm{m}$ & $(60,160)$ \\
\hline Number of nodes & 120 \\
\hline Initial energy $/ \mathrm{J}$ & $0.5 \sim 1$ \\
\hline Data pack /bit & 2000 \\
\hline Control package /bit & 50 \\
\hline $\mathrm{p}$ & 0.05 \\
\hline Etx $/ \mathrm{nJ} / \mathrm{bit}$ & 50 \\
\hline Erx $/ \mathrm{nJ} / \mathrm{bit}$ & 50 \\
\hline Eda $/ \mathrm{nJ} / \mathrm{bit}$ & 5 \\
\hline Efs $/ \mathrm{pJ} / \mathrm{bit} / \mathrm{m} 2$ & 10 \\
\hline Emp $/ \mathrm{pJ} / \mathrm{bit} / \mathrm{m} 4$ & 0.0013 \\
\hline
\end{tabular}

\subsection{Determine the optimal number of clusters}

For the AOCRP routing protocol, the optimal number of clusters needs to be determined first. According to the size of the wireless sensor network measurement area, the total number of nodes, the distance from the node to the base station and other parameters are obtained through theoretical calculation or through simulation experiments to obtain the optimal number of clusters. The $120 \mathrm{~m} \times 80 \mathrm{~m}$ area of the wireless sensor network is divided into 1, 2, 3, 4, 6 and 8 cluster areas respectively, and the experiment is carried out under the environment with uniform node distribution. The experimental curves are shown in Fig. 3 and Fig. 4. 


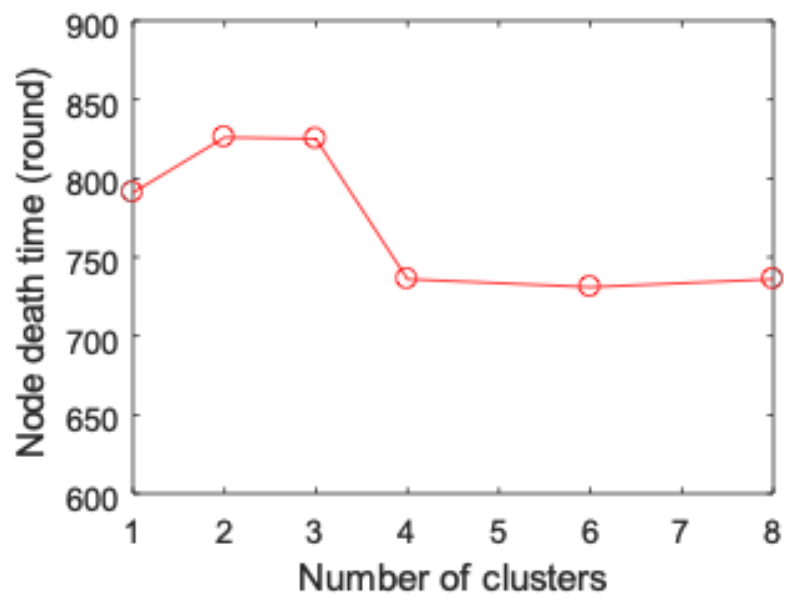

Fig. 3. $30 \%$ node death curve of AOCRP algorithm

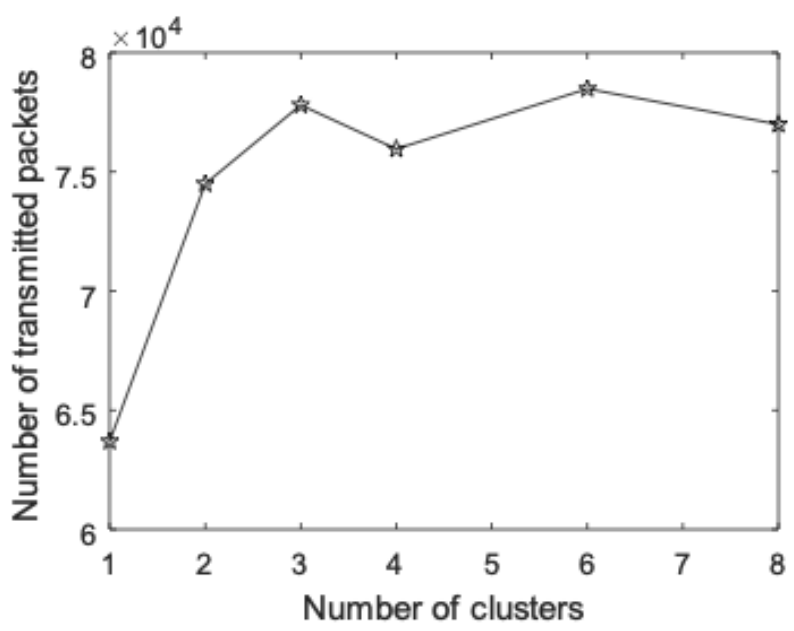

Fig. 4. Packet transmission curve of AOCRP algorithm

It can be seen from Fig. 3 and Fig. 4 that, under a given area size and total number of nodes, considering $30 \%$ of the node dead time and the data throughput of the wireless sensor network, the three cluster areas are optimal. Figure 5 is a schematic diagram of division into three cluster regions. 


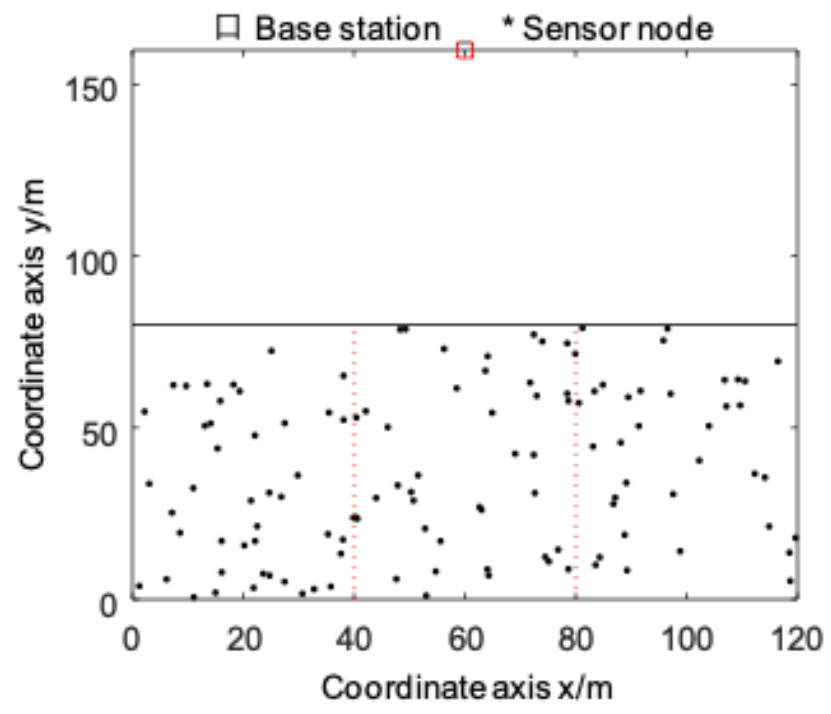

Fig. 5. Schematic diagram of division into 3 cluster regions

\subsection{Analysis of simulation results}

The EH-LEACH, DEEC, and AOCRP routing protocols were each tested 100 times. In each experiment, 120 sensor nodes were randomly generated, as shown in Figure 2. The initial energy of each node is heterogeneous. When conducting the AOCRP routing protocol experiment, first determine the optimal cluster number $k$ (in the experimental environment given in this paper, find $k=3$ ), and the entire region is divided into $\mathrm{k}$ identical cluster areas. The balance factor in the election function f(i) takes $a=0.6$. The node survival number of the 100-pass experiment and the average value of the data packets sent to the base station were taken as experimental results, and a comparison curve was drawn. In each experiment, the nodes of the EH-LEACH, DEEC, and AOCRP routing protocols are identically distributed, and the initial energy of the same node is the same, that is, the operating environments of the three clustering routing protocols are exactly the same. In our experiments, the effects of random factors such as wireless channel interference and signal collisions were ignored.

Network life cycle. The number of surviving nodes in the whole network changes with the running time of the network to reflect the length of the network life cycle, and thereby act as an important indicator that reflects the efficiency of the algorithm. The images of the number of surviving nodes over time by different algorithms can more intuitively compare the changes of the network life cycle in different algorithms.

The comparison of the number of surviving nodes for three different routing protocols is shown in Figure 6. The first node of the AOCRP routing protocol dies at 3.94 times of the EH-LEACH routing protocol, and the $30 \%$ node dies at 2.65 times the EHLEACH routing protocol. The 50\% node death time is EH-LEACH routing protocol. 2.07 times. The first node of the AOCRP protocol dies 2.05 times of the DEEC routing protocol, $30 \%$ of the nodes die 1.54 times of the DEEC routing protocol, and $50 \%$ of 
the nodes die 1.58 times of the DEEC routing protocol. This shows that the AOCRP protocol distributes the energy loss more evenly among all nodes, avoiding the premature death of a single node due to excessive energy loss. The death of the network can be considered when the number of dead nodes in the wireless sensor network occupies a certain proportion. As can be seen from Figure 6, the AOCRP routing protocol extends the superior performance of the wireless sensor network life cycle.

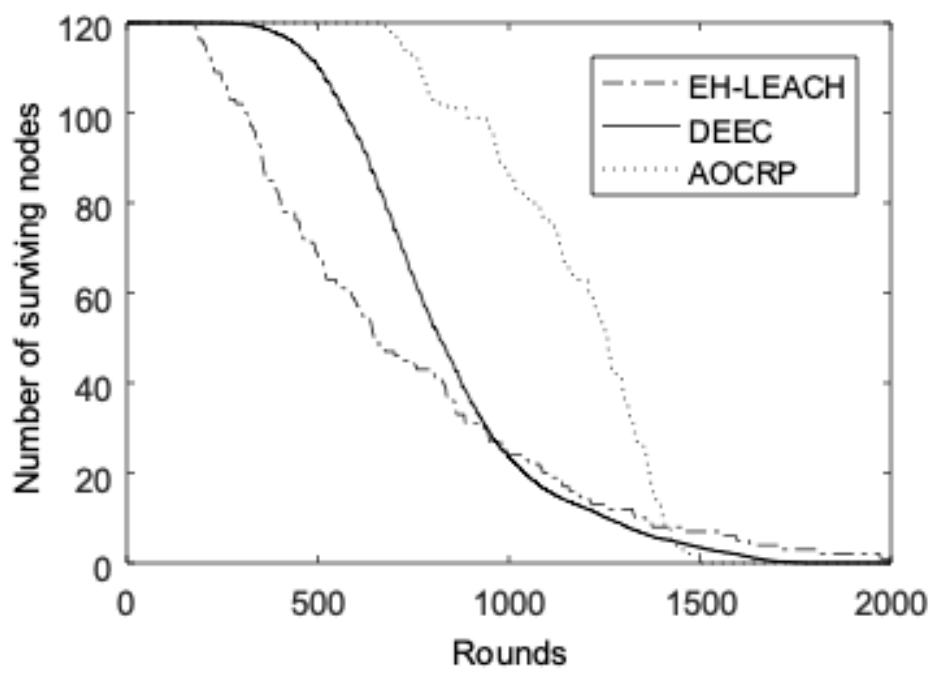

Fig. 6. Comparison of the number of surviving nodes in three different routing protocols

The AOCRP protocol improves the life cycle of the network by improving the cluster head election mechanism, making the cluster heads more evenly distributed, adjusting the number of nodes in each cluster to be the same in each round, and reducing the premature death of some nodes due to fast energy consumption.

Network throughput. Network throughput refers to the amount of data sent by each node in the network during communication with the base station.

Figure 7 is a comparison of network throughput of three different routing protocols, that is, the relationship between data packets received by the base station and time. The total number of packets sent by the AOCRP protocol is 1.89 times that of EH-LEACH and 1.48 times that of DEEC. This is because the cluster head election of the EHLEACH routing protocol does not consider the energy of the nodes. The cluster heads of the elections are unevenly distributed. Some cluster heads communicate with the base station at a long distance, causing their load to be too large and the energy to be exhausted quickly, resulting in premature death. The number of nodes in the network is reduced, and the coverage area is reduced. Although the cluster head election of the DEEC routing protocol considers the node energy, the cluster heads of the election are not evenly distributed. Some cluster heads are far away from the base station, and the energy is quickly exhausted and causes premature death. This further illustrates that AOCRP can save network energy consumption and improve sensor energy utilization. 


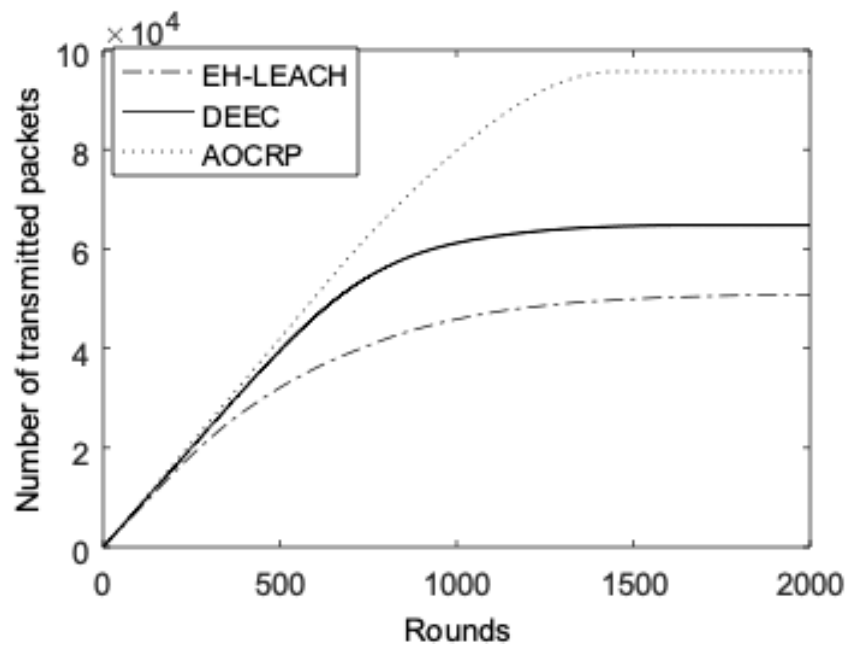

Fig. 7. Comparison of network throughput of three different routing protocols

The AOCRP protocol optimizes the clustering and automatically adjusts the number of nodes in each cluster area to make the cluster heads more evenly distributed, thereby reducing the communication load of some nodes, and also reducing too much of energy consumption by some of the nodes, so that the nodes can send more data packets leading to higher energy utilization of the sensor nodes.

\section{Conclusion}

The theoretical analysis and simulation results show that the proposed AOCRP routing protocol is superior to the classical EH-LEACH routing protocol and DEEC routing protocol in network lifetime and network throughput performance. The AOCRP routing protocol has an optimized and fixed number of clusters, automatically adjusts the number of nodes in each cluster, and makes the number of nodes in each cluster the same, and the cluster head distribution is relatively uniform. The AOCRP routing protocol overcomes the many shortcomings of the LEACH routing protocol and a series of improved routing protocols, such as the random generation of cluster heads, inconsistent number of nodes in each cluster, and uneven distribution. Since the AOCRP routing protocol keeps the cluster size the same, the cluster head distribution is relatively more uniform, which equalizes the node energy loss, prolongs the life cycle of the wireless sensor network, and increases the network throughput.

\section{$7 \quad$ Acknowledgment}

Project Source: National Natural Science Foundation of China (61562038); National Natural Science Foundation of China (61562037); Natural Science Foundation of Jiangxi Province of China (20171BAB202026) 


\section{$8 \quad$ References}

[1] Yick J, Mukherjee B, Ghosal D. (2008) Wireless sensor network survey. Computer Networks, 52(12):2292-2330. https://doi.org/10.1016/j.comnet.2008.04.002

[2] Bi Xiao-jun, Diao Peng-fei. (2017) Routing and clustering algorithm heterogeneous wireless sensor networks based on gravitational search algorithm. Control and Deci-sion, 32(3):563569.

[3] Badia-Melis R, Ruiz-Garcia L, Garcia-Hierro J, et al. (2015) Refrigerated Fruit Storage Monitoring Combining Two Different Wireless Sensing Technologies: RFID and WSN.Sensors, 15(3):4781-4795. https://doi.org/10.3390/s150304781

[4] Brar G S, Rani S, Chopra V, et al. (2016) Energy Efficient Direction-Based PDORP Routing Protocol for WSN. IEEE Access, 4:3182-3194. https://doi.org/10.1109/ACCESS. 2016.2576475

[5] Zhu J, Liu J, Hai Z, et al. (2016) Research on Routing Protocol Facing to Signal Con-flicting in Link Quality Guaranteed WSN. Wireless Networks, 22(5):1739-1750. https://doi.org/10.1007/s11276-015-1053-6

[6] Heinzelman W B, Chandrakasan A P, Balakrishnan H, et al. (2002) An application-specific protocol architecture for wireless microsensor networks. IEEE Transactions on Wireless Communications, 1(4):660-670 https://doi.org/10.1109/TWC.2002.804190

[7] Lin Z X, Wei L, Yu H B, et al. (2012) Survey of transmission scheduling methods in wireless sensor networks. Journal on Communications, 33(5):143-157.

[8] Huang Li-xiao, Wang Hui, Yuan Li-yong, et al (2017) Improved LEACH protocol algorithm for WSN based on energy balance and high efficiency. Journal on Communi-cations, 38(Z2):164-169.

[9] Qing L, Zhu Q X, Wang M W. (2006) Design of a distributed energy-efficient cluster-ing algorithm for heterogeneous wireless sensor networks. Computer Communica-tions, 17(3): 481-489.

[10] Li An-chao, Chen Gui-fen. (2017) An Improved clustering routing algorithm for ener-gy heterogeneous wireless sensor networks. Chinese Journal of Sensors and Actua-tors, 30(11):1712-1718.

[11] Gupta S K, Jana P K. (2015) Energy efficient clustering and routing algorithms for wireless sensor networks: GA based approach. Wireless Personal Communications, 83(3):2403-2423 https://doi.org/10.1007/s11277-015-2535-7

[12] Kuila P, Jana P K. (2014) Energy efficient clustering and routing algorithms for wire-less sensor networks: Particle swarm optimization approach. Engineering Applica-tions of Artificial Intelligence, 33(5):127-140 https://doi.org/10.1016/j.engappai.2014.04.009

[13] Wei C J, Yang J J, Zhang Z M. (2013) A distributed energy-efficient clustering routing protocol for wireless sensor networks. Chinese Journal of sensors and Actuators, 26(7):1014-1018

[14] Wang W. (2014) Design of routing protocol in long distance band type wireless sen-sor network. Computer Engineering, 40(3):132-136.

[15] Li Cheng-fa, Chen Gui-Hai, Ye Mao. (2007) An uneven cluster-based routing protocol for wireless sensor networks. Chinese Journal of Computers, 30(1):27-36

[16] Wang Jin-wei, Sun Hua-zhi, Sun De-bing. (2011) Research on the number of optimal cluster heads of wireless sensor networks based on energy consumption. Transducer and Microsystem Technologies, 30(7):45-47, 50 


\section{Authors}

Hu Zhongdong, Wang Zhendong, Wang Junling, and Zeng Ting are with the School of Information Engineering, Jiangxi University of Science and Technology, Ganzhou, 341000, China (jxhzd@163.com).

Article submitted 29 July 2018. Resubmitted 16 September 2018. Final acceptance 11 November 2018. Final version published as submitted by the authors. 